Pacific

Journal of

Mathematics

A PROPERTY OF FREE ENTROPY

S.T. Belinschi and H. Bercovici 


\title{
A PROPERTY OF FREE ENTROPY
}

\author{
S.T. Belinschi AND H. Bercovici
}

\begin{abstract}
We show that the restriction on the uniform norms of approximating matricial microstates can be removed when defining free entropy.
\end{abstract}

\section{Introduction.}

Denote by $\mathfrak{M}_{k}$ the algebra of complex $k \times k$ matrices, and by $\tau_{k}$ the normalized trace on $\mathfrak{M}_{k}$, i.e., $\tau_{k}(A)=\frac{1}{k} \operatorname{Tr}(A)$ for $A \in \mathfrak{M}_{k}$. Consider for each $k$ a standard Gaussian Hermitian random matrix $X_{k}$. Thus, if $E$ denotes expected value, $E \tau_{k}\left(X_{k}\right)=0$ and $E \tau_{k}\left(X_{k}^{2}\right)=1$. It was shown by E. Wigner [9] that, as $k \rightarrow \infty, X_{k}$ tends in distribution to a semicircular law, i.e., the limits

$$
\mu_{p}=\lim _{k \rightarrow \infty} E \tau_{k}\left(X_{k}^{p}\right)
$$

exist, and they can be calculated as

$$
\mu_{p}=\frac{1}{2 \pi} \int_{-2}^{2} t^{p} \sqrt{4-t^{2}} d t
$$

for $p=1,2, \ldots$. If we have several independent standard Gaussian Hermitian random matrices $\left(X_{k}(i)\right)_{i=1}^{n}$, D. Voiculescu [4] proved that, as $k \rightarrow \infty$, these sets of variables converge in distribution to a free semicircular family. Briefly, this means that given indices $i_{j} \in\{1,2, \ldots, n\}$ such that $i_{j} \neq i_{j+1}$ for $j=1,2, \ldots, m-1$, and given positive integers $p_{1}, p_{2}, \ldots, p_{m}$, the limit

$$
\lim _{k \rightarrow \infty} E \tau_{k}\left(X_{k}\left(i_{1}\right)^{p_{1}} X_{k}\left(i_{2}\right)^{p_{2}} \ldots X_{k}\left(i_{m}\right)^{p_{m}}\right)
$$

exists, and

$$
\lim _{k \rightarrow \infty} E \tau_{k}\left[\left(X_{k}\left(i_{1}\right)^{p_{1}}-\mu_{p_{1}}\right)\left(X_{k}\left(i_{2}\right)^{p_{2}}-\mu_{p_{2}}\right) \ldots\left(X_{k}\left(i_{m}\right)^{p_{m}}-\mu_{p_{m}}\right)\right]=0 .
$$

It is natural to look for large deviation principles associated with these limit laws. For this purpose (and also with motivation from information theory and statistical physics) Voiculescu introduced in [6] (cf. also [5]) the notion of free entropy. The original definition of free entropy, which will be reviewed below, involves a bound $R>0$ on the operator norm of approximating matricial microstates, and this may perhaps obscure its significance for large deviations. It is our purpose here to show that this bound can be removed - roughly speaking, one can set $R=\infty$ in the 
definition of free entropy. This result applies to other notions of free entropy which appeared subsequently (see for instance [7] for free entropy in the presence of additional variables, [8] for free entropy using an ultrafilter, and [3] for free entropy of a nonselfadjoint variable). We will only provide the proof for the original quantity $\chi$ defined in [6], but it should be obvious how the argument applies in the other situations.

It should be noted that a large deviation theorem for Wigner's result has been proved by G. Ben Arous and A. Guionnet [1], where the natural topology of weak convergence of probability measures on the real line is used. The rate function is closely related with free entropy. For several variables, a thorough study of large deviations was undertaken by T. Cabanal Duvillard and A. Guionnet [2]. The rate function they determine is related with another version of free entropy (microstate free).

\section{The main result.}

For the remainder of this note we fix a positive integer $n$. We will denote by $I$ the collection of all multiindices $\alpha=\left(\alpha_{1}, \alpha_{2}, \ldots, \alpha_{m}\right)$ with $m \geq 1$ and $\alpha_{j} \in$ $\{1,2, \ldots, n\}$ for all $j=1,2, \ldots, m$. In other words, $I=\bigcup_{m=1}^{\infty}\{1,2, \ldots, n\}^{m}$. A multiindex of the form $(\alpha, \alpha, \ldots, \alpha)$ will also be denoted $\alpha^{m}$. We consider the space $\mathbb{S}$ consisting of all families $(\mu(\alpha))_{\alpha \in I}$ of complex numbers indexed by $I$. The space $\mathbb{S}$ will be endowed with the topology of componentwise convergence.

Consider now a tracial $W^{*}$-probability space $(\mathfrak{A}, \tau)$. That is, $\mathfrak{A}$ is a von Neumann algebra, and $\tau$ is a normal trace state on $\mathfrak{A}$. We will write $\mathfrak{A}^{\text {sa }}$ for the space of selfadjoint elements of $\mathfrak{A}$. Given an $n$-tuple $X=$ $\left(X_{1}, X_{2}, \ldots, X_{n}\right) \in\left(\mathfrak{A}^{\mathrm{sa}}\right)^{n}$, its distribution $\mu_{X} \in \mathbb{S}$ is defined by

$$
\mu_{X}(\alpha)=\tau\left(X_{\alpha}\right)
$$

where $X_{\alpha}=X_{\alpha_{1}} X_{\alpha_{2}} \ldots X_{\alpha_{m}}$ for $\alpha=\left(\alpha_{1}, \alpha_{2}, \ldots, \alpha_{m}\right) \in I$. This notation applies in particular to $n$-tuples of selfadjoint matrices in $\mathfrak{M}_{k}$. Voiculescu's entropy measures the extent to which the distribution of $X$ can be approximated by distributions of the form $\mu_{A}$ with $A \in\left(\mathfrak{M}_{k}^{\mathrm{sa}}\right)^{n}$. Note first that $\mathfrak{M}_{k}^{\mathrm{sa}}$ is a real Hilbert space with the Hilbert-Schmidt norm $\|A\|_{2}=\operatorname{Tr}\left(A^{2}\right)$, and $\lambda_{k}$ will denote the corresponding Lebesgue measure (i.e., a cube whose sides form an orthonormal basis has measure equal to one). On the space $\left(\mathfrak{M}_{k}^{\mathrm{sa}}\right)^{n}$ we have the product measure $\lambda_{k}^{\otimes n}$.

Given $X \in\left(\mathfrak{A}^{\text {sa }}\right)^{n}$, and a neighborhood $U$ of $\mu_{X}$ in $\mathbb{S}$, we set

$$
\Gamma(X ; k, U)=\left\{A \in\left(\mathfrak{M}_{k}^{\mathrm{sa}}\right)^{n}: \mu_{A} \in U\right\} .
$$

Given in addition a positive number $R$,

$$
\Gamma_{R}(X ; k, U)=\left\{A \in \Gamma(X ; k, U):\left\|A_{j}\right\|<R \text { for all } j\right\} .
$$


We can then define the quantities

$$
\chi_{R}(X ; U)=\liminf _{k \rightarrow \infty}\left[\frac{1}{k^{2}} \log \lambda_{k}^{\otimes n}\left(\Gamma_{R}(X ; k, U)\right)+\frac{n}{2} \log k\right],
$$

and

$$
\chi_{R}(X)=\inf _{U} \chi_{R}(X ; U),
$$

where $U$ runs over a neighborhood base of $\mu_{X}$ in $\mathbb{S}$. Finally, the free entropy is defined as

$$
\chi(X)=\sup _{R>0} \chi_{R}(X) .
$$

We also set

$$
\chi_{\infty}(X ; U)=\liminf _{k \rightarrow \infty}\left[\frac{1}{k^{2}} \log \lambda_{k}^{\otimes n}(\Gamma(X ; k, U))+\frac{n}{2} \log k\right],
$$

and $\chi_{\infty}(X)=\inf _{U} \chi_{\infty}(X ; U)$. This quantity was introduced in the concluding remarks of $[\mathbf{6}]$, where other possible definitions of free entropy are discussed briefly. The inequalities

$$
\chi_{R}(X) \leq \chi(X) \leq \chi_{\infty}(X)
$$

are obvious for $R>0$, and Proposition 2.4 of [6] states that $\chi_{R}(X)=\chi(X)$ if $R$ is sufficiently large; $R>\max _{j}\left\|X_{j}\right\|$ will suffice. Our main result is as follows:

Proposition 2.1. For every $X \in\left(\mathfrak{A}^{\mathrm{sa}}\right)^{n}$ we have $\chi(X)=\chi_{\infty}(X)$.

The proof of this result is a refinement of the proof of Proposition 2.4 in [6]. We begin by considering the diffeomorphism $f$ of the real line onto $(-2,2)$ defined by $f(t)=t$ for $t \in[-1,1], f(t)=2-\frac{1}{t}$ for $t>1$, and $f(t)=-2-\frac{1}{t}$ for $t<-1$. Observe that $f^{\prime}$ does not have any local minimum, and therefore

$$
\frac{f(s)-f(t)}{s-t} \geq \min \left\{f^{\prime}(s), f^{\prime}(t)\right\} \geq f^{\prime}(s) f^{\prime}(t)
$$

for all $s$ and $t$. The function $F_{n}:\left(\mathfrak{M}_{k}^{\mathrm{sa}}\right)^{n} \rightarrow\left(\mathfrak{M}_{k}^{\mathrm{sa}}\right)^{n}$ defined by

$$
F_{n}\left(A_{1}, A_{2}, \ldots, A_{n}\right)=\left(f\left(A_{1}\right), f\left(A_{2}\right), \ldots, f\left(A_{n}\right)\right)
$$

is also differentiable, and we need to estimate the Jacobian determinant $\left(J F_{n}\right)(A)$. Since

$$
\left(J F_{n}\right)(A)=\left(J F_{1}\right)\left(A_{1}\right)\left(J F_{1}\right)\left(A_{2}\right) \ldots\left(J F_{1}\right)\left(A_{n}\right),
$$

it suffices to do this in one variable. As pointed out in [6], if $A$ is a $k \times k$ matrix with eigenvalues $\mu_{1}, \mu_{2}, \ldots, \mu_{k}$, we have

$$
\left(J F_{1}\right)(A)=\left(\prod_{i \neq j} \frac{f\left(\mu_{i}\right)-f\left(\mu_{j}\right)}{\mu_{i}-\mu_{j}}\right) \cdot \prod_{i=1}^{k} f^{\prime}\left(\mu_{i}\right) .
$$


By the estimate for difference quotients shown above,

$$
\begin{aligned}
\left(J F_{1}\right)(A) & \geq\left(\prod_{i \neq j} f^{\prime}\left(\mu_{i}\right) f^{\prime}\left(\mu_{j}\right)\right) \cdot \prod_{i=1}^{k} f^{\prime}\left(\mu_{i}\right) \\
& =\prod_{i=1}^{k} f^{\prime}\left(\mu_{i}\right)^{2 k-1}=\prod_{\left|\mu_{i}\right|>1} \mu_{i}^{-2(2 k-1)} .
\end{aligned}
$$

Denoting $\log ^{+}(t)=\max \{\log t, 0\}$, we obtain

$$
\begin{aligned}
\log \left(J F_{1}\right)(A) & \geq-2(2 k-1) \sum_{i=1}^{k} \log ^{+} \mu_{i} \\
& =-2 k(2 k-1) \frac{1}{k} \sum_{i=1}^{k} \log ^{+} \mu_{i} \\
& =-2 k(2 k-1) \tau_{k}\left(\log ^{+}|A|\right) .
\end{aligned}
$$

We have therefore proved the following estimate:

Lemma 2.2. Given $A=\left(A_{1}, A_{2}, \ldots, A_{n}\right) \in\left(\mathfrak{M}_{k}^{\mathrm{sa}}\right)^{n}$, we have

$$
\left(J F_{n}\right)(A) \geq \exp \left[-2 k(2 k-1) \sum_{j=1}^{n} \tau_{k}\left(\log ^{+}\left|A_{j}\right|\right)\right] \text {. }
$$

Note for further use that, for a selfadjoint $k \times k$ matrix $A, \tau_{k}\left(\log ^{+}|A|\right)$ can be estimated in terms of the moments $\tau_{k}\left(A^{2 p}\right), p \geq 1$. In fact, $\log ^{+} t=$ $\frac{1}{2 p} \log ^{+} t^{2 p} \leq \frac{1}{2 p} t^{2 p}$, and therefore

$$
\tau_{k}\left(\log ^{+}|A|\right) \leq \frac{1}{2 p} \tau_{k}\left(A^{2 p}\right) .
$$

We need one more ingredient.

Lemma 2.3. Let $X \in\left(\mathfrak{A}^{\mathrm{sa}}\right)^{n}$ satisfy $\max _{j}\left\|X_{j}\right\|<1$, and let $U$ be a neighborhood of $\mu_{X}$ in $\mathbb{S}$. There exists a neighborhood $V$ of $\mu_{X}$ in $\mathbb{S}$ such that

$$
F_{n}(\Gamma(X ; k, V)) \subset \Gamma_{2}(X ; k, U) \text { for all } k \text {. }
$$

Proof. Clearly it suffices to prove the lemma for neighborhoods of the form

$$
U=\left\{\mu \in \mathbb{S}:\left|\mu(\alpha)-\tau\left(X_{\alpha}\right)\right|<\varepsilon\right\},
$$

where $\alpha=\left(\alpha_{1}, \alpha_{2}, \ldots, \alpha_{m}\right) \in I$ and $\varepsilon>0$ are fixed. Using the Hölder inequality

$$
\left\|A_{\alpha}\right\|_{1} \leq\left\|A_{\alpha_{1}}\right\|_{m}\left\|A_{\alpha_{2}}\right\|_{m} \ldots\left\|A_{\alpha_{m}}\right\|_{m}
$$

we see that it is sufficient to choose $V$ so that, for all $A \in \Gamma(X ; k, V)$, we have $\left|\tau_{k}\left(A_{\alpha}\right)-\tau\left(X_{\alpha}\right)\right|<\varepsilon / 2,\left\|A_{j}\right\|_{m} \leq 1$, and $\left\|A_{j}-f\left(A_{j}\right)\right\|_{m}<\varepsilon / 2 m$ for 
$j=1,2, \ldots, n$. Choose a number $r<1$ so that $r>\left\|X_{j}\right\|$ for all $j$, and choose an even integer $q>m$ such that $r^{q / m}<\varepsilon / 2 m$. Define next

$$
V=\left\{\mu \in \mathbb{S}:\left|\mu(\alpha)-\tau\left(X_{\alpha}\right)\right|<\frac{\varepsilon}{2} \text { and }\left|\mu\left(j^{q}\right)\right|<r^{q} \text { for } j=1,2, \ldots, n\right\} ;
$$

recall that $j^{q}$ denotes the $q$-multiindex with all entries equal to $j$. Consider now $A \in \Gamma(X ; k, V)$, and note that the inequalities $\left|\tau_{k}\left(A_{\alpha}\right)-\tau\left(X_{\alpha}\right)\right|<\varepsilon / 2$ are obviously satisfied. Also,

$$
\left\|A_{j}\right\|_{m} \leq\left\|A_{j}\right\|_{q}=\tau\left(A_{j}^{q}\right)^{1 / q} \leq r<1 .
$$

Finally, if $\mu_{1}, \mu_{2}, \ldots, \mu_{k}$ are the eigenvalues of $A_{j}$,

$$
\begin{aligned}
\left\|A_{j}-f\left(A_{j}\right)\right\|_{m} & \leq\left(\frac{1}{k} \sum_{\left|\mu_{j}\right| \geq 1}\left|\mu_{j}\right|^{m}\right)^{1 / m} \leq\left(\frac{1}{k} \sum_{\left|\mu_{j}\right| \geq 1}\left|\mu_{j}\right|^{q}\right)^{1 / m} \\
& \leq\left(\tau_{k}\left(A_{j}^{q}\right)\right)^{1 / m} \leq r^{q / m}
\end{aligned}
$$

and this quantity is less than $\varepsilon / 2 m$.

Proof of Proposition 2.1. It suffices to prove the proposition in case $\left\|X_{j}\right\|<$ 1 for all $j$. From the results of $[6]$ we know that $\chi_{2}(X)=\chi(X)$, and clearly $\chi_{2}(X) \leq \chi_{\infty}(X)$. To prove the opposite inequality $\chi_{2}(X) \geq \chi_{\infty}(X)$, let $U$ be a neighborhood of $\mu_{X}$ in $\mathbb{S}$, and let $V$ be the neighborhood of $\mu_{X}$ furnished by Lemma 2.3, i.e., $F_{n}(\Gamma(X ; k, V)) \subset \Gamma_{2}(X ; k, U)$ for all $k \geq 1$. Given a positive integer $p$, we may also assume that $\tau_{k}\left(A_{j}^{2 p}\right) \leq 1$ whenever $A=\left(A_{1}, A_{2}, \ldots, A_{n}\right) \in \Gamma(X ; k, V)$. It follows then from Lemma 2.2 (and the remark following its statement) that

$$
\left(J F_{n}\right)(A) \geq \exp \left[-2 k(2 k-1) \frac{n}{2 p}\right]
$$

for all $A \in \Gamma(X ; k, V)$. Since the function $F_{n}$ is one-to-one, we deduce that

$$
\begin{aligned}
\lambda_{k}^{\otimes n}\left(\Gamma_{2}(X ; k, U)\right) & \geq \lambda_{k}^{\otimes n}\left(F_{n}(\Gamma(X ; k, V))\right) \\
& \geq \exp \left[-2 k(2 k-1) \frac{n}{2 p}\right] \lambda_{k}^{\otimes n}(\Gamma(X ; k, V)) .
\end{aligned}
$$

Therefore

$$
\begin{aligned}
& \frac{1}{k^{2}} \log \lambda_{k}^{\otimes n}\left(\Gamma_{2}(X ; k, U)\right)+\frac{n}{2} \log k \\
& \geq \frac{1}{k^{2}} \log \lambda_{k}^{\otimes n}(\Gamma(X ; k, V))+\frac{n}{2} \log k-\left(2-\frac{1}{k}\right) \frac{n}{p},
\end{aligned}
$$

and as $k \rightarrow \infty$ this yields

$$
\chi_{2}(X ; U) \geq \chi_{\infty}(X ; V)-\frac{2 n}{p} .
$$


Since $p$ is arbitrary, we deduce that $\chi_{2}(X ; U) \geq \chi_{\infty}(X ; V) \geq \chi_{\infty}(X)$, and the proof is concluded by taking the infimum over $U$.

We remark that a suitable modification of the above proof yields directly that $\chi_{\infty}(X)=\chi_{R}(X)$ if $\left\|X_{j}\right\|<R$. One needs an appropriate version of the function $f$, and that is easily constructed.

\section{References}

[1] G. Ben Arous and A. Guionnet, Large deviations for Wigner's law and Voiculescu's noncommutative entropy, Probab. Theory Related Fields, 108 (1997), 517-542, MR 98i:15026, Zbl 0954.60029.

[2] T. Cabanal Duvillard and A. Guionnet, Large deviations upper bounds for the laws of matrix-valued processes and non-commutative entropies, Ann. Probab., 29 (2001), 1205-1261, MR 2003a:60040.

[3] P. Śniady, Inequality for Voiculescu's free entropy in terms of Brown measure, Int. Math. Res. Notices, (2003), 51-64, CMP 1935566.

[4] D. Voiculescu, Limit laws for random matrices and free products, Invent. Math., 104 (1991), 201-220, MR 92d:46163, Zbl 0736.60007.

[5] - The analogues of entropy and Fisher's information measure in free probability, I, Comm. Math. Phys., 155 (1993), 71-92, MR 94k:46137, Zbl 0781.60006.

[6] The analogues of entropy and Fisher's information measure in free probability, II, Invent. Math., 1118 (1994), 411-440, MR 96a:46117, Zbl 0820.60001.

[7] _ The analogues of entropy and Fisher's information measure in free probability. III. The absence of Cartan algebras, Geom. Funct. Anal., 6 (1996), 172-199, MR 96m:46119, Zbl 0856.60012.

[8] _ A strengthened asymptotic freeness result for random matrices with applications to free entropy, Internat. Math. Res. Notices, (1998), 41-63, MR 2000d:46080, Zbl 0895.60004.

[9] E. Wigner, On the distribution of the roots of certain symmetric matrices, Ann. Math., 67 (1958), 325-327, MR 20 \#2029, Zbl 0085.13203.

Received May 6, 2002 and revised June 20, 2002. The second author was supported in part by a grant from the National Science Foundation.

Institute OF MATHEMATiCS

ROMANIAN ACADEMY

P. O. Box 1-764

Bucharest RO-70700

ROMANIA

E-mail address: sbelinsc@indiana.edu

Mathematics Department

INDIANA UNIVERSITY

Bloomington, IN 47405

E-mail address: bercovic@indiana.edu 